\title{
A Matrix Ensemble with a Preferential Basis and its Application to Disordered Metals and Insulators
}

\author{
Jean-Louis Pichard ${ }^{(1)}$ and Boris Shapiro ${ }^{(1,2)}$ \\ (1), C.E.A., Service de Physique de l'Etat Condensé, Centre d'Études de Saclay, \\ 91191 Gif-sur-Yvette Cédex, France \\ (2), Department of Physics, Technion - Israel Institute of Technology, \\ 32000 Haifa, Israel
}

\begin{abstract}
The standard ensembles of the random matrix theory are invariant under change of basis. For non interacting electrons in disordered systems, this invariance is broken and deviations from the random matrix theory predictions occur, especially for strong disorder. We consider a generalization of the standard ensembles which includes a preferential basis and which gives rise to a "screening" of the logarithmic pairwise interaction between energy levels. In the unitary case, we recover a mathematically tractable distribution of energy levels first introduced by Gaudin. This simplified model provides a qualitative description of level statistics in the metal, insulator and at the mobility edge, which only depends on the dimensionless conductance $g$.
\end{abstract}

PACS numbers: $02.50 .+\mathrm{s}, 05.45 .+\mathrm{b}, 72.15 . \mathrm{Rn}$ 
The standard Gaussian ensembles in random matrix theory [?] can be defined as ensembles of matrices with independent (apart from the symmetries) normally distributed elements $H_{i j}$. For instance, the Gaussian orthogonal ensemble (G.O.E.) consists of real symmetric $N \times N$-matrices $\mathbf{H}$ with $\left\langle H_{i j}\right\rangle=0,\left\langle H_{i i}^{2}\right\rangle=1,\left\langle H_{i j}^{2}\right\rangle=$ $1 / 2, i \neq j$. The angular brackets denote averaging over the ensemble. An important property of the G.O.E is its invariance under orthogonal transformations, i.e. the absence of any preferential basis. The invariance property makes possible a rather complete analytical treatment of the ensemble. The statistics of the eigenvalues $E_{\alpha}$ is given by the charge distribution of a one-dimensional Coulomb gas [?] with pairwise logarithmic interaction $\ln \left|E_{\alpha}-E_{\beta}\right|$. This universal interaction, with no parameters at all, leads to universal level statistics. For instance it can be shown [?,?] that the density-density correlations of the energy levels depend only on this universal pairwise interaction.

In disordered electronic systems, the invariance under change of basis is broken. At least for non interacting electrons, this is particularly clear for strong disorder where the eigenstates become localized in space. For example, in a tight-binding Anderson Hamiltonian, in the limit of strong disorder, the eigenvectors become localized on individual sites of the lattice, i. e. all matrices of the ensemble become diagonal in the site representation and the eigenvalues become independent from each other. But even for weak disorder (metal) the G.O.E. description breaks down for energy intervals larger than the Thouless energy [?,?] $E_{c} \approx \hbar / \tau$, where $\tau$ is the time required for a wave packet to get across the sample. Using the Coulomb gas analogy one can say that the pairwise logarithmic interaction gets screened at distances larger than $E_{c}$ [?]. More generally, defining a dimensionless conductance as $g=E_{c} / \Delta$, where $\Delta$ is the average level spacing, and relying on the one-parameter scaling theory of localization [?], one can assume that level statistics is controlled by the single scaling parameter $g$. In the $3 d-$ metal, $g$ increases linearly as a function of the system size so that many consecutive levels follow the G.O.E. statistics in the thermodynamic limit. But for a system at the mobility edge, the conductance is scale invariant $g=g_{0} \approx 1$ and the G.O.E. ceases to be applicable already for the nearest neighbor levels [?,?]. In the insulator $g$ becomes exponentially small and energy levels become essentially uncorrelated (Poisson Statistics).

In this letter we consider a simplified one-parameter ensemble, with a preferential basis, which provides a qualitative description of level statistics in the metal, insulator and at the mobility edge. Let us first consider an ensemble of real symmetric matrices with independently and normally distributed elements, satisfying $\left\langle H_{i i}^{2}\right\rangle=1$ and $\left\langle H_{i j}^{2}\right\rangle=1 /(2(1+\mu))$ for all $i>j$. Thus, the probability density to find a matrix in the volume element 
$\prod_{i \leq j}^{N} d H_{i j}$ is given by:

$$
P\left(\left\{H_{i j}\right\}\right) \propto \exp \left[-\frac{1}{2} \sum_{i=1}^{N} H_{i i}^{2}-(1+\mu) \sum_{i<j}^{N} H_{i j}^{2}\right] .
$$

When $\mu$ is changing from 0 to $\infty$, for any fixed $N$, the ensemble changes from G.O.E. to an ensemble of diagonal matrices with independently distributed eigenvalues. In particular, the distribution $P(S)$ of the nearest neighbor level spacing will change from the WignerDyson distribution to the Poisson one. Such a crossover has been demonstrated numerically by Rosenzweig and Porter many years ago [?]. More recently Leyvraz and Seligman [?] have computed the two-point level correlation function in the limit of large $\mu$ (i. e. for a Poisson ensemble with a small G.O.E. correction).

Since $\mathbf{H}$ is real symmetric, we have $\mathbf{H}=\mathbf{R} \cdot \mathbf{H}_{d} \cdot \mathbf{R}^{T}$ where $\mathbf{R}$ is a $N$-dimensional orthogonal transformation, $\mathbf{R}^{T}$ its transpose and $\mathbf{H}_{d}=\operatorname{diag}\left(E_{1}, \ldots, E_{N}\right)$. Transforming Eq. (1) to eigenvalue-eigenvector variables enables us to identify the term inducing the eigenvalueeigenvector correlations by which the level repulsion get suppressed. These observations, supplemented by an exact solution of the $N=2$ case, pave the way for a derivation of a maximum entropy ensemble with a preferential basis. The transformation from $H_{i j}$ to the eigenvalues $E_{\alpha}$ and the eigenvectors $R_{\alpha i}(\alpha, i=1, \ldots, N)$, as described for instance in Ref. [?], leads to

$W\left(\left\{E_{\alpha}, R_{\alpha i}\right\}\right) \propto \prod_{\alpha<\beta=1}^{N}\left|E_{\alpha}-E_{\beta}\right| \cdot \exp \left[-\frac{1}{2} \sum_{\alpha=1}^{N} E_{\alpha}^{2}-\frac{\mu}{2} \sum_{\alpha<\beta}^{N}\left(E_{\alpha}-E_{\beta}\right)^{2} \sum_{i=1}^{N} R_{\alpha, i}^{2} R_{\beta i}^{2}\right]$.

The function $W$ describes the probability density to find a matrix $\mathbf{H}$ with eigenvalues in the volume element $\prod_{\alpha=1}^{N} d E_{\alpha}$ and eigenvectors in the volume element $d \tau_{R}$ corresponding to the invariant measure of the orthogonal group [?]. $R_{\alpha i}$ is the $i$-th component of the eigenvector $\mid \alpha>$ in some basis $\mid i>$, e. g. in the site basis of a tightbinding Hamiltonian. The combination $\sum_{i=1}^{N} R_{\alpha i}^{2} R_{\beta i}^{2}$ is not invariant under a change of the basis and, for large $\mu$, the eigenvectors are forced to stay close to the vectors $\mid i>$ of the preferential basis. In the large $\mu$ limit, the matrix $\mathbf{R}$ is close to the unit matrix $\mathbf{1}$ and can be writen as $\mathbf{R} \approx \mathbf{1}+\mathbf{A}$ where the antisymmetric real $N \times N-$ matrix A depends on the $N(N-1) / 2$ "angles" needed to parametrize a rotation in a $N$-dimensional space. In this limit, one has

$$
\sum_{i=1}^{N} R_{\alpha, i}^{2} R_{\beta, i}^{2} \approx 2 A_{\alpha, \beta}^{2}
$$

and one can integrate Eq. (2) over the variables $A_{\alpha, \beta}$ from $-\infty$ to $\infty$ with the measure $\prod_{\alpha<\beta} d A_{\alpha \beta}$. This integration eliminates the level repulsion factors $\left|E_{\alpha}-E_{\beta}\right|$ 
and one ends up with the uncorrelated level distribution $\exp \left[-\left(\sum_{a=1}^{N} E_{\alpha}^{2}\right)\right]$. It is important to realize that this result holds strictly for $\mu \rightarrow \infty$. For any finite $\mu$, levels with sufficiently close energies will eventually repel each other. The "small angle" approximation, under which Eq. (3) was derived, becomes invalid when $\left|E_{\alpha}-E_{\beta}\right|$ is smaller than $E_{\mu}=1 / \sqrt{\mu}$. The energy $E_{\mu}$ is here playing the role of $E_{c}$ and the G.O.E. description is approximately valid only for energy intervals smaller than $E_{\mu}$. It is worthwhile to note that, when $\mu$ is gradually increased, the G.O.E. statistics first breaks down close to the edges of the Wigner semicircle level density and only later in the middle of the energy band. This is due to the low density of levels at the edges, as compared with the density in the middle of the band.

This qualitative discussion is supported by exact results for the $N=2$ case where an orthogonal matrix can be writen as:

$$
\mathbf{R}=\left(\begin{array}{ll}
\cos \theta & \sin \theta \\
-\sin \theta & \cos \theta
\end{array}\right)
$$

and Eq.(2) reduces to:

$$
W\left(E_{1}, E_{2}, \theta\right) \propto|s| \exp \left[-\frac{E_{1}^{2}+E_{2}^{2}}{2}-\mu s^{2} \sin ^{2} \theta \cos ^{2} \theta\right]
$$

where $s=E_{1}-E_{2}$. Integration over $\theta$, with the invariant measure $d \theta /(2 \pi)$, produces the following expression for the level distribution:

$P\left(E_{1}, E_{2}\right) \propto|s| \exp \left(-\frac{E_{1}^{2}+E_{2}^{2}}{2}\right) \cdot \exp \left(-\frac{\mu s^{2}}{8}\right) \cdot I_{0}\left(\frac{\mu s^{2}}{8}\right)$,

where $\mu s^{2}=\left(s / E_{\mu}\right)^{2}$. The function $f(x)=$ $\exp (-x) \cdot I_{0}(x)$ approaches unity for $x \rightarrow 0$ and decreases as $1 / \sqrt{x}$ for $x>>1$. Thus for $s<<E_{\mu}$ one has the G.O.E. level statistics, while for $s>>E_{\mu}$ the repulsion factor $|s|$ is cancelled and one obtains two independent levels.

If one integrates Eq. (5) over $E_{2}$, for a fixed value $E_{1}=E$, one obtains the probability distribution $F(E, \theta)$ to find an eigenstate $(\cos \theta, \sin \theta)$ with an energy $E$ :

$F(E, \theta) \propto \exp \left(-E^{2}\right) \cdot \frac{1}{p}\left[1+E \sqrt{\frac{\pi}{4 p}} \exp \left(\frac{E^{2}}{4 p}\right) \cdot \operatorname{erf}\left(\frac{E}{2 \sqrt{p}}\right)\right]$

where $p=\frac{1}{2}+\mu \sin ^{2} \theta \cos ^{2} \theta$. For $\mu=0$ this expression is independent of $\theta$, i. e. , at any energy, all eigenvectors occur with equal probability. However, for finite (small) $\mu$ an approximatelly uniform distribution for $\theta$ occurs only for $E<E_{\mu}$, whereas in the opposite limit the distribution is strongly peaked at small angles, $\theta<\left(E_{\mu} / E\right)$. 
In the general $N \times N$ case there are $N(N-1) / 2$ independent angles, on which the eigenvectors in Eq. (2) depend, and the integration over these angles does not seem to be possible. To circumvent this difficulty we propose a simplified ensemble in which the angles are replaced by angle-like variables $A_{\alpha \beta}$. These variables form a real antisymmetric $N \times N$-matrix and, in contrast with the true angles, are allowed to vary from $-\infty$ to $+\infty$. The introduction of such variables is motivated by the following observation. Let us look again at Eq. (5) and at the function $f(x)=\exp (-x) \cdot I_{0}(x)$ which appears after integration over $\theta$. To understand better the behavior of this function, let us note that, for $s>E_{\mu}$, the expression $\sin ^{2} \theta \cos ^{2} \theta$ in Eq. (5) can be replaced by $\theta^{2}$ and the integration over $\theta$ can be done from $-\infty$ to $+\infty$ (rather than from 0 to $2 \pi$ ). This leads to the $1 / \sqrt{x}$ behavior of the function $f(x)$, for large $x$. In the opposite case $\left(s<<E_{\mu}\right)$, the term $\mu s^{2} \sin ^{2} \theta \cos ^{2} \theta$ in Eq. (7) can be omitted and integration over $\theta$ gives a constant. Thus the behavior for both large and small values of $\mu s^{2}$ can be qualitatively reproduced by introducing, instead of the true angle $\theta$, our angle-like variable $-\infty<A<+\infty$ and by replacing the factor $\mu s^{2} \sin ^{2} \theta \cos ^{2} \theta$ by a simpler expression $A^{2}+\mu s^{2} A^{2}$. Integration over $A$ then becomes trivial and gives $(1+x)^{-1 / 2}$. This function replaces the more complicated function $f(x)$ and it smoothly interpolates between small $\mu s^{2}\left(s<<E_{\mu}\right.$, G. O. E.) and large $\mu s^{2}$ ( $s>>E_{\mu}$, independent levels).

These considerations lead us to the formulation of a simplified maximum entropy ensemble. A maximum entropy ensemble corresponds to the "most random" ensemble which fulfills some expectation values (constraints), and is rigorously defined [?] from a maximization of the information entropy associated to the probability density $P\left(\left\{E_{\alpha}, A_{\alpha \beta}\right\}\right)$, given these constraints. Our ensemble results from three constraints: $\left\langle\operatorname{tr} \mathbf{H}^{2}\right\rangle=$ $C_{1},\left\langle\sum_{\alpha<\beta}^{N} A_{\alpha \beta}^{2}\right\rangle=C_{2}$, and $\left\langle\sum_{\alpha<\beta}^{N}\left(E_{\alpha}-E_{\beta}\right)^{2} A_{\alpha \beta}^{2}\right\rangle=$ $C_{3}$. The first constraint is the standard one employed in the studies of the gaussian ensembles. The second constraint introduces a preferential basis with respect to which the "angles" $A_{\alpha \beta}$ are defined. The real antisymmetric matrix $\mathbf{A}$ replaces the orthogonal matrix $\mathbf{R}$ of the more "microscopic" ensemble defined by Eq. (2). For small $\mathbf{A}$, the two matrices are related by $\mathbf{R}=\mathbf{1}+\mathbf{A}$. For large $\mathbf{A}$, the relation with the matrix $\mathbf{R}$ becomes vague, i. e. : we are no longer able to describe the eigenvectors of $\mathbf{H}$. But, as has been already mentioned, occurence of large angles with a significant probability means that levels statistics is close to G.O.E. and the integration over the angles amounts to a constant. Finally, the third constraint describes coupling between the eigenvalues and the angle-like variables $A_{\alpha \beta}$. The specific choice of the coupling, namely $\sum_{\alpha<\beta}\left(E_{\alpha}-E_{\beta}\right)^{2} A_{\alpha \beta}^{2}$, is motivated by the considerations presented above.

The three constraints lead to a maximum entropy en- 
semble [?] with three Lagrange multipliers. Two of the multipliers fix the scale of energy and of the angle-like variables $A_{\alpha \beta}$, so that the final expression for the probability density $P\left(\left\{E_{\alpha}, A_{\alpha \beta}\right\}\right)$ contains one parameter $\mu$ and has the form:

$P\left(\left\{E_{\alpha}, A_{\alpha \beta}\right\}\right) \propto \prod_{\alpha<\beta}^{N}\left|E_{\alpha}-E_{\beta}\right| \cdot \exp \left[-\frac{1}{2} \sum_{\alpha=1}^{N} E_{\alpha}^{2}-\sum_{\alpha<\beta}^{N} A_{\alpha \beta}^{2}-\mu \sum_{\alpha<\beta}^{N}\left(E_{\alpha}-E_{\beta}\right)^{2} A_{\alpha \beta}^{2}\right]$.

The factors $\left|E_{\alpha}-E_{\beta}\right|$ come from the volume element in the energy-angle space. Let us also mention that the unitary case, i. e. an ensemble of hermitian matrices, can be treated in complete analogy with the orthogonal case considered above. The real antisymmetric matrix $\mathbf{A}$ is then replaced by an anti-hermitian matrix. Integrating out the angle-like variable, and setting $E_{\mu}=\sqrt{1 / \mu}$, we obtain the following expression for $P\left(E_{1}, \ldots, E_{N}\right)$, which encompases both the orthogonal $(\boldsymbol{\beta}=1)$ and the unitary $(\boldsymbol{\beta}=2)$ case:

$P\left(E_{1}, \ldots, E_{N}\right) \propto \exp \left[-\frac{1}{2} \sum_{\alpha=1}^{N} E_{\alpha}^{2}\right] \prod_{\alpha<\alpha^{\prime}}^{N}\left[\frac{\left(E_{\alpha}-E_{\alpha^{\prime}}\right)^{2}}{E_{\mu}^{2}+\left(E_{\alpha}-E_{\alpha^{\prime}}\right)^{2}}\right]^{\frac{\beta}{2}}$

It is interesting that the same expression (but without the exponential factor) was obtained by Yukawa [?] who used a rather different approach. Yukawa considered the "dynamics" of eigenvalues $E_{\alpha}(\lambda)$ under a change of some parameter $\lambda$ in the hamiltonian. In his formulation $\lambda$ plays the role of time and $E_{\alpha}$ 's are canonical coordinates. Some additional auxiliary canonical variables are introduced and a Hamilton function, describing the level dynamics, is derived. The main assumption is that, in the $\lambda \rightarrow \infty$ limit, the system equilibrates to a Gibbs distribution with two parameters which are the analogs of energy and angular momentum. Integration over the auxiliary variables yields Eq. (9) for $P\left(E_{1}, \ldots, E_{N}\right)$. Another earlier piece of work that should be mentioned in this context is the work of Gaudin [?] who introduced the function given by Eq. (9) (but without the exponential factor), for $\boldsymbol{\beta}=2$, as a possible generalization of the Dyson Coulomb gas [?]. This model was introduced mostly for its mathematical tractability rather than from "microscopic" consideration. Gaudin, and subsequently Forrester [?], gave a rather complete analytical treatment of the model (n-point correlation functions, nearest level spacing distribution...).

Since one is mainly interested in the local level statistics, i. e. energy scales within which the average density of levels does not change, the factor in Eq. (9) coming from the constraint $C_{1}$ is not essential. The point we wish to make is that Eq. (9) provides a simplified description of the level statistics for any degree of disorder, which allows us to incorporate the scaling picture of localization 
in a random matrix description. Many qualitative properties of the level statistics are recovered in the metal, in the insulator and at the mobility edge, and the system size dependence can enter through a single scaling parameter. As discussed above, $E_{\mu}$ should be identified with $E_{c}$, which in turn is related to the dimensionless conductance $g=E_{c} / \Delta$. Then, one can write Eq. (9) as

$$
P\left(\epsilon_{1}, \ldots, \epsilon_{N}\right) \propto \exp \left[-\frac{\beta}{2} \sum_{\alpha<\alpha^{\prime}} \ln \left(1+\frac{g^{2}}{\left(\epsilon_{\alpha}-\epsilon_{\alpha^{\prime}}\right)^{2}}\right)\right],
$$

where $\epsilon_{\alpha}=E_{\alpha} / \Delta$.

$P\left(\epsilon_{1}, \ldots, \epsilon_{N}\right)$ corresponds now to the distribution of classical charges interacting via a "screened" pairwise potential at a temperature $\boldsymbol{\beta}^{-1}$. Only for $\left|\epsilon_{\alpha}-\epsilon_{\alpha^{\prime}}\right|<<g$, we recover the standard logarithmic interaction. For $\left|\epsilon_{\alpha}-\epsilon_{\alpha^{\prime}}\right|>g$, the pairwise interaction decays as $\left|\epsilon_{\alpha}-\epsilon_{\alpha^{\prime}}\right|^{-2}$. The weakness of this model is that it does not contain the space dimension $d$ (in a $d$-dimensional metal the long range part of the pairwise interaction decreases [?] with a $d$-dependent exponent and this remains true at the mobility edge [?]). This weakness can be traced to the fact that all off-diagonal matrix elements in Eq. (1) (or all the angle-like variables in the maximum entropy ensemble (8)) are treated on the same footing which corresponds to long range hopping in a tight-binding hamiltonian. The nature of the localization of the eigenstates in those models is also somewhat particular. In the microscopic hamiltonian (1), for large $\mu$, the main part of the wave function amplitude is concentrated at some given site, while the remaining part is scatterered in a totally random way over the other sites. This differs from an exponentially Anderson localized state, characterized by a localization length $\xi$. However our model leads us to a mathematically tractable level distribution which improves the standard statistics of the orthogonal and unitary ensembles, and contains many qualitative features derived in microscopic models.

The free parameter $g$ of the model can vary with $N$ in a prescribed way. Metallic behavior corresponds to $g$ increasing with $N$. G.O.E. (G.U.E.) then holds for larger and larger consecutive sequences of levels. Insulating behavior corresponds to $g$ decreasing with $N$. In the large N-limit the function $P\left(E_{1}, \ldots, E_{N}\right)$ reduces essentially to a constant: only for very close levels $g^{2}$ in Eq. (10) should be kept and the level repulsion can be felt.

The most interesting case is when $g=g_{0} \approx 1$ is scale invariant. This corresponds to the mobility edge situation. We only discuss the distribution $P(s)$ for nearest neighbor spacings, at the mobility edge. This function has been studied numerically by Shklovskiı̌ et al [?]. They suggest that $P(s)$, for the orthogonal case, is a universal function which is linear for small $s$ and decreases exponentially for large $s$ ( for the unitary case the small 
$s$ behavior should be $s^{2}$, instead of $s$ ). It is interesting to compare the results of Ref. 9, for the Anderson model, with the analytical results based on Eq. (11), for $g=g_{0} \approx 1$. Such results are available, due to the work of Gaudin and Forrester. It turns out [?] that, for $\boldsymbol{\beta}=2$, $P(s)$ is indeed proportional to $s^{2}$, for small $s$. For large $s$, it decreases as $s^{\delta} \exp (-\gamma s)$ where $\gamma$ and $\delta$ are numbers of order one.

In conclusion, the description of level statistics in disordered systems, for arbitrary degree of disorder, requires departure from the standard Gaussian ensembles and introduction of more general matrix ensembles, with a preferential basis. One such possibility is explored in this paper. Using microscopic considerations, as well as a maximal entropy principle, we have derived a one-parameter ensemble (in agreement with the scaling theory of localization) with a preferential basis. We have shown that it gives a qualitative description of the level statistics in the metal, the insulator and at the mobility edge. This description can be summarized by a very simple Coulomb gas analogy, as pointed out in Refs 16-17. The energy level statistics becomes formally identical to the Gibbs distribution of classical charges interacting via an infinite range logarithmic pairwise potential. The temperature takes the usual values $(\boldsymbol{\beta}=1,2)$, depending on the system symmetries. The charges are free to move on a line parallel to a metal wall - a mathematically tractable distribution in the unitary case, first introduced by Gaudin. The metallic wall induces mirror charges which "screen" the pairwise interaction of the levels. This distribution contains a single parameter, the distance between the line and the wall, which we identify as the dimensionless conductance $(g / 2)$.

Useful discussions with I. Affleck, C.W.J. Beenakker, J.-P. Bouchaud, F. Leyvraz and P. A. Mello are gratefully acknowledged. This work was supported in part by the Fund for Promotion of Research at the Technion.

[1] M. L. Mehta, Random Matrices (Academic, New York, 1991, $2^{\text {nd }}$ edition).

[2] F. J. Dyson, J. Math. Phys. 3, 140 (1962).

[3] C. W. J. Beenakker, Phys. Rev. Lett. 70, 1155 (1993).

[4] E. Brezin and A. Zee, Nucl. Phys. 402, 613 (1993) and preprint NSF-ITP-93-124.

[5] B.L. Al'tshuler and B.I. Shklovskiǔ, Zh. Eksp. Teor. Fiz. 91, 220 (1986) [Sov. Phys. JETP 64, 127 (1986)].

[6] N. Argaman, Y. Imry, and U. Smilansky, Phys. Rev. B 47, 4440 (1993).

[7] R. Jalabert, J.-L. Pichard and C. W. J. Beenakker, Europhys. Lett. 24, 1 (1993).

[8] E. Abrahams, P. W Anderson, D. C. Licciardello and T. V. Ramakrishnan, Phys. Rev. Lett. bf 42, 673 (1979). 
[9] B.I. Shklovskiı̆, B. Shapiro, B. R. Sears, P. Lambrianides and H. B. Shore, Phys. Rev. B 47, 11487 (1993).

[10] V. E. Kravtsov, I. V. Lerner, B. L. Altshuler and A. G. Aronov, preprint.

[11] N. Rosenzweig and C. E. Porter, Phys. Rev. 120, 1698 (1960).

[12] F. Leyvraz and T. H. Seligman, J. Phys. A. 23, 1555 (1990).

[13] C. E. Porter, Statistical Theory of Spectra: Fluctuations, 1965 (Academic, New York), ed. by C. E. Porter.

[14] R. Balian, Nuovo Cimento, 57, 183 (1968).

[15] T. Yukawa, Phys. Rev. Lett 17, 1883 (1985) and Phys. Lett. A 116, 227 (1986).

[16] M. Gaudin, Nucl. Phys. 85, 545 (1966).

[17] P. J. Forrester, Phys. Lett. A 173, 355 (1993). 